\title{
GBT and VLA investigation of the ionized gas towards the Galactic center
}

\author{
M. J. Royster and F. Yusef-Zadeh \\ Department of Physics \& Astronomy and CIERA, \\ Northwestern University, Evanston, IL, 60208, USA \\ email: mjroyster@u.northwestern.edu
}

\begin{abstract}
We report results of a study of the ionized gas towards the Galactic center with radio recombination lines at $\mathrm{cm}$ wavelengths. Both the Green Bank Telescope and the Very Large Array were utilized to probe the kinematics of the ionized gas on a global scale for both diffuse and discrete sources within the inner $2.0^{\circ} \times 0.5^{\circ}(l \times b)$. A diffuse $\sim 0 \mathrm{~km} \mathrm{~s}^{-1}$ gas, a thermal flux continuum fraction exceeding $40 \%$, and an asymmetry where $\sim 70 \%$ of the ionized gas is found at positive Galactic longitudes are the preliminary results briefly discussed here.
\end{abstract}

\section{Introduction}

The distribution of ionized gas in the Galactic center (GC) has been probed on a scale of a few parsecs using hydrogen radio recombination lines (RRLs) and with [NeII] (Roberts \& Goss 1993; Zhao et al. 2010; Irons et al. 2012). However, a global picture of the ionized gas in the inner $250 \mathrm{pc}$ has yet to be carried out. Towards this end, we have utilized the Green Bank Telescope (GBT) and the Very Large Array (VLA) to study the diffuse ionized component with RRLs at $\mathrm{cm}$ wavelengths.

The GBT observations include a complete survey of the inner $300 \times 75 \mathrm{pc}(l \times b)$ of the GC with spatial and spectral resolutions of $73^{\prime \prime}$ and $<1 \mathrm{~km} \mathrm{~s}^{-1}$ respectively. A frequency-switched observing scheme was utilized with velocity ranges of $\sim 500 \mathrm{~km} \mathrm{~s}^{-1}$. RRLs from H86 $\alpha$ - H91 $\alpha(10.1 \mathrm{GHz}-8.58 \mathrm{GHz})$ were observed simultaneously and coadded in velocity space after re-sampling to a common grid. Although RRLs typically suffer from a low line-to-continuum (LTC) ratio, the ability to co-add multiple transitions in order to increase sensitivity is unique to the radio and $\mathrm{mm}$ regime.

\section{Results}

Kinematics of diffuse emission: The large-scale kinematics are presented here as a longitude-velocity image (see Figure 1). Prominent GC features are clearly distinguished (and labeled) in Figure 1. Coincident with Sgr B2/B1 and extending from $l=0.7^{\circ}-0.5^{\circ}$ is a roughly $-150 \mathrm{~km} \mathrm{~s}^{-1}$ forbidden feature previously not detected in past RRL studies of the Sgr B clouds (e.g. Mehringer et al. 1993). At negative longitudes, significant emission is seen from $-150 \mathrm{~km} \mathrm{~s}^{-1}$ to $+50 \mathrm{~km} \mathrm{~s}^{-1}$ within the 'Sgr A - C Bridge.' This includes the thermal sources that constitute $\mathrm{Sgr} \mathrm{C}$ itself, where multiple components at $0,-50$, and $-150 \mathrm{~km} \mathrm{~s}^{-1}$ are detected along the line-of-sight. We have already acquired further position-switched GBT observations to confirm these kinematic features.

There is also a clear asymmetry in ionized flux about $l=0.0^{\circ}$ with $\sim 70 \%$ found at positive longitudes. This is similar to the molecular asymmetry where for example $60 \%$ of the flux is found at positive longitudes in the CS line (Tsuboi et al. 1999) within the inner 2 degrees.

A pervasive $\sim 0 \mathrm{kms}^{-1}$ diffuse ionized gas is also detected and evident in both the 


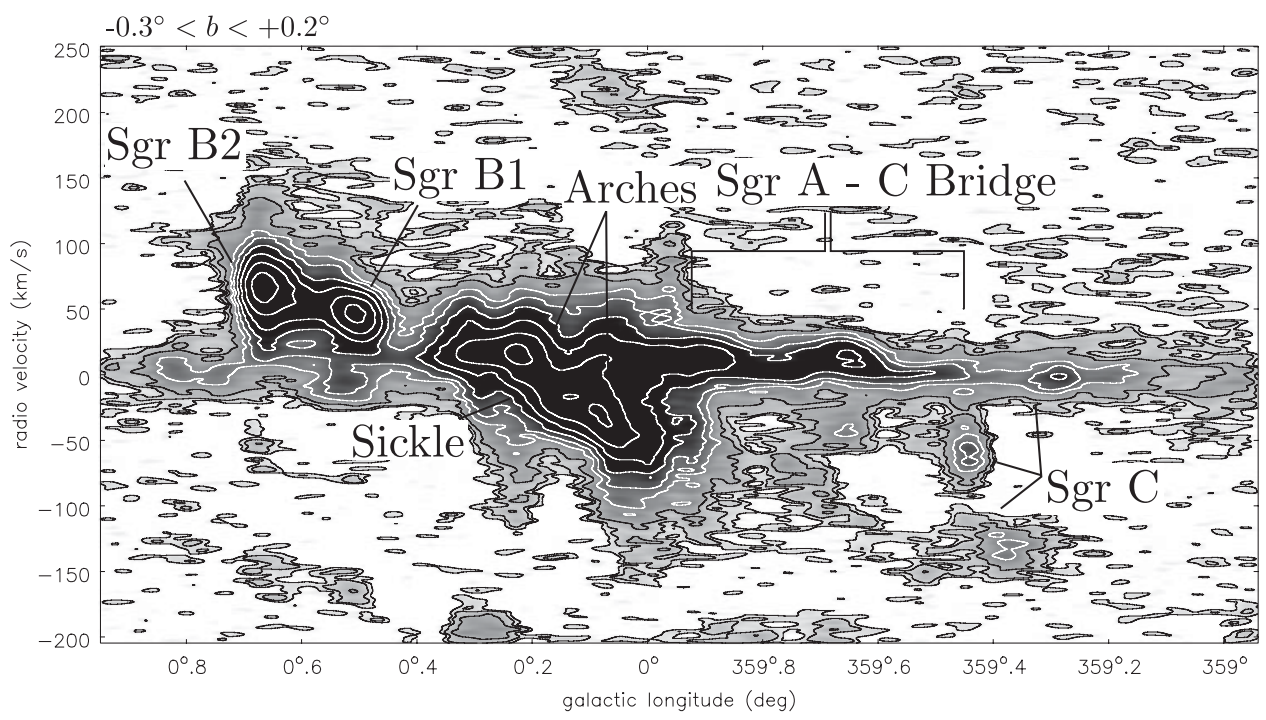

Figure 1. Longitude-velocity image of the mapped region of $\mathrm{H} 86 \alpha-\mathrm{H} 91 \alpha$ summed from $-0.3^{\circ}$ to $+0.2^{\circ}$ in Galactic latitude. The image was spatially and spectrally convolved with a $90^{\prime \prime} \times 4.5$ $\mathrm{km} \mathrm{s}^{-1}$ 'beam' and has an RMS of $\sigma=31.5$ mJy. Black contours are at $1 \sigma, 2 \sigma$, and $4 \sigma$; white contours begin at $8 \sigma$ and increase in factors of 1.4 .

longitude-velocity and velocity-latitude diagrams at all observed longitudes and latitudes. Inspection unveils typical center velocities, $\left|v_{\mathrm{LSR}}\right|<10 \mathrm{~km} \mathrm{~s}^{-1}$ and line widths of $30 \mathrm{~km} \mathrm{~s}^{-1}<v_{\text {F н н }}<60 \mathrm{~km} \mathrm{~s}^{-1}$. From the velocity-latitude diagram, the aforementioned diffuse gas appears to favor higher velocities at negative latitudes. This diffuse ionized gas shares many traits with the extended low density warm ionized medium observed at low frequency $(<2 \mathrm{GHz})$, which is believed to originate from the outer envelopes of HII regions along the line-of-sight (Anantharamaiah 1985).

Global properties: The Galactic center hosts a significant amount of nonthermal flux that can contaminate recombination LTC estimates. To determine local thermodynamic equilibrium (LTE) electron temperatures $\left(\mathrm{T}_{e}\right)$, we assumed a $30 \%$ thermal flux fraction (Law et al. 2008). We find $\mathrm{T}_{e}$ (LTE) consistently within the $5000 \mathrm{~K}-10000 \mathrm{~K}$ range where line emission is $2 \sigma$ or above.

On the other hand, RRL emission can be used to estimate the contribution of nonthermal emission with the assumption of an LTE electron temperature. Thus, by taking a canonical $\mathrm{T}_{e}$ value of $7500 \mathrm{~K}$ we determined the distribution of thermal continuum flux. The contribution at negative longitudes is found to be consistent with that previously seen by Law et al. (2008) of $\sim 30 \%$. However, the thermal contribution from Sgr B1/B2 as well as HiI regions in the thermal Arches and Arches counterpart significantly increase the thermal continuum fraction to $\sim 45 \%$ at positive longitudes.

\section{References}

Anantharamaiah, K. R. 1985, JApA 6, 203

Irons, W. T., Lacy, J. H., \& Richter, M. J. 2012, ApJ 755, 901

Law, C. J., Yusef-Zadeh, F., Cotton, W. D., \& Maddalena, R. J. 2008, ApJS 177, 255

Mehringer, M. M., Palmer, P. P., Goss, W. M., \& Yusef-Zadeh, F. 1993, ApJ 412, 684

Roberts, D. A. \& Goss, W. M. 1993, ApJS 86, 133

Tsuboi, M., Handa, T., \& Ukita, N. 1999, ApJS 120, 1

Zhao, J., Blundell, R., Moran, J. M., et al. 2010, ApJ 723, 1097 